\title{
Exploranation: A New Science Communication Paradigm
}

Anders Ynnerman, J onas Löwgren and Lena Tibell

The self-archived postprint version of this journal article is available at Linköping University Institutional Repository (DiVA):

http:/ / urn.kb.se/ resolve?urn=urn:nbn:se:liu:diva- 149382

N.B.: When citing this work, cite the original publication.

Ynnerman, A., Löwgren, J ., Tibell, L., (2018), Exploranation: A New Science Communication Paradigm, IEEE Computer Graphics and Applications, 38(3), 13-20.

https:// doi.org/ 10.1109/ MCG.2018.032421649

Original publication available at:

https:/ / doi.org/ 10.1109/ MCG.2018.032421649

Copyright: Institute of Electrical and Electronics Engineers (IEEE)

http:/ / www.ieee.org/ index.html

(C) 2018 IEEE. Personal use of this material is permitted. However, permission to reprint/ republish this material for advertising or promotional purposes or for creating new collective works for resale or redistribution to servers or lists, or to reuse any copyrighted component of this work in other works must be obtained from the IEEE. 


\section{Exploranation:}

\section{A New Science Communication Paradigm}

\author{
Anders Ynnerman \\ Linköping University \\ Jonas Löwgren \\ Linköping University \\ Lena Tibell \\ Linköping University \\ Editors: \\ Beatriz Sousa Santos \\ bss@ua.pt \\ Ginger Alford \\ alfordg@trinityvalleyschool \\ .org
}

\author{
Science communication is facing a paradigm shift, based on the \\ convergence of exploratory and explanatory visualization. In this article, we
} coin the term exploranation to denote the way in which visualization

methods from scientific exploration can be used to communicate results and how methods in explanatory visualization can enrich exploration.

The visual language spans borders of knowledge, experience, age, gender, and culture. This arguably makes visualization the most effective form of expression in science communication. Visual science communication is facing a paradigm shift caused by the rapid pace of digitalization and widespread availability of visual data analysis tools and

computing resources. Until now there has been a clear division between visualization enabling effective data analysis leading to scientific discovery (exploratory visualization) and visual representations used to explain and communicate science to a general audience (explanatory visualization). To denote the confluence of exploration and explanation, we coin the neologism exploranation, as a combination of the two terms.

The underpinning trend behind the paradigm shift is based on the emerging strong synergy between exploratory and explanatory visualization driven by two connected facts:

- Science communication can now be directly based on real, large-scale and/or complex scientific data-i.e., the same data that researchers have gathered and explored for scientific discovery.

- Advanced visualization and interaction methodology has reached a high level of maturity and can be used on standard GPUs, making it possible to use the same visualization approaches in both exploratory and explanatory visualization.

The use of visual exploranation approaches in science communication is still in its infancy. At the Norrköping Visualization Center in Sweden, a center with research units and public facilities with 150,000 visitors per year, we have, over the past decade, worked on developing a range of visualization experiences for the general public and in particular for schoolchildren. To do this, we've been using various technical platforms and storytelling approaches in interactive data-driven visualization.

It is through this work that we have identified the disruptive change in science communication that we are facing, and we have started to systematically approach the challenges involved in bringing interactive data visualization to the public. These challenges are reaching into the foundation of not only technical areas such as data access and representations, real-time data analysis, visual representations, and rendering and interaction paradigms, but also research areas such as storytelling in interactive media and design for visual learning. On a fundamental level, there is also a need to develop a theory and understanding of how visual literacy is established in interactive exploration. Additionally, we need ways to measure user engagement or involvement and the corresponding effect on learning outcomes in exploranation scenarios.

On a general note, it can be added that over the past decades, visualization has proven itself to be a highly versatile tool, being deployed in a range of applications spanning across academic and commercial domains, enabling scientific breakthroughs, improving industrial workflows, creating new business models, etc. However, one question often asked is why the visualization research community has not been more actively involved in the development of some of the most widespread visualization services such as Google Earth, 
services that reach a large-scale general public. One of the answers to this question may be found in the emphasis visualization research has had on exploratory visualization used by domain experts. It is our hope that the convergence of exploratory and explanatory approaches will lead to the increased and widespread impact of interactive data visualization approaches.

In this article we feature three scenarios, providing examples and showcasing different flavors of how we have worked with exploranation applications. We then conclude by providing a set of empirically verified design recommendations and a summary from the visual-learning perspective.

\section{EXPLORANATION SCENARIOS}

One of the underpinning strategies in our work to bring interactive data visualization to public spaces is based on the need to produce functioning installations that are robust enough to endure interactions with large numbers of visitors per year. This entails going the extra mile in terms of robustness, reliability, and extracting feedback from user experiences of the software. We have seen this as an enabling factor in gaining insights into the special demands on interactive data visualization that science communication makes.

\section{From Medical Researchers to Visitors at the British Museum}

Medical-imaging modalities are generating increasingly large amounts of data. In a single full-body scan from a computerized-tomography scanner, approximately 20,000 high-resolution data slices can be generated in just a few seconds. Interactive direct volume rendering (DVR) of this data has become one of the main analysis tools. In our research on DVR, we developed a range of methods to deal with data sizes ${ }^{1}$ and methods to improve the quality and speed of volumetric lighting. ${ }^{2}$ We also implemented our software on largescale multitouch tables. ${ }^{3}$

All this work was targeting medical-domain experts, clinicians, and medical students. However, we soon realized that the interactive exploration of medical data on touch tables raised a tremendous amount of interest among the general public and, in particular, visitors to the Visualization Center. At the Center, we had placed one touch table with sample datasets and a simplified interface with presets and limited interaction, together with a narrative evolving with the help of information hotspots. In essence, this insight was the starting point for the concept of exploranation - that the same data and underlying methods can be used for both experts in professional tasks and lay audiences in public settings. Interacting at a large touch table affords the formation of temporary social structures around objects of interest. Interfaces combining overall narrative structures with scaffolded, self-directed examination facilitate the integration of explanatory and exploratory modes of inquiry (more on this to follow).

Formal evaluations of our public installations in museums and science centers show that audience engagement is consistently very high. More importantly, the installation of touch tables to explore the insides of interesting artifacts in museums (for an example, see Figure 1) in fact increases the proportion of visitors studying the actual artifacts. For a comprehensive example, refer to our work with the Gebelein Man mummy at the British Museum. ${ }^{4}$

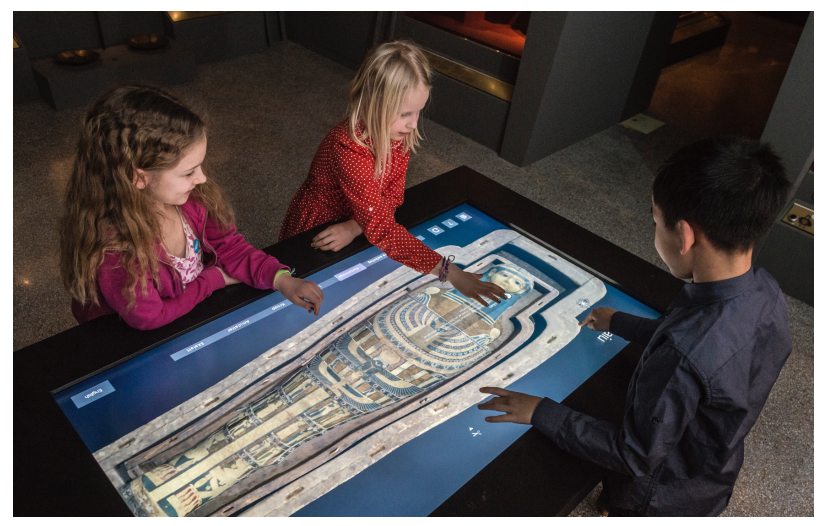

Figure 1. Children at the Mediterranean Museum in Stockholm using our visualization table to interactively explore a data visualization of an ancient Egyptian mummy. 


\section{Perceiving the Forces in the Microcosmos}

For 30 to 40 years, visualization has been one of the most common communication methods in cell- and molecular-biology research to analyze large amounts of data - for example, the coordinates of thousands of atoms in large dynamic molecules and the rate constants for their interactions. The objects are very small $(\mathrm{nm})$, their shapes do not have a direct resemblance to macroscopic visual metaphors, and the underlying processes are complex. Interactions at the molecular level are of fundamental importance for all aspects of life and involve the participation of many weak forces. These interactions are dynamic, complex, and stochastic in nature but lead to highly regulated processes. In fact, they are highly counterintuitive, ${ }^{5,6}$ especially when described in text.

To explore the added value of multimodal interaction in visual learning and to enable improved understanding of molecular interactions, we created a haptic application named MolDock (see Figure 2). Molecular coordinates for a protein are imported from the Protein Data Bank (PDB files), and a 3D model of a protein and a corresponding ligand is created. The protein-ligand interaction is then mapped to force feedback on the haptic device, using our toolkit for proxy-based volumetric haptic rendering. ${ }^{7}$ The application is implemented on a Phantom device with collocated stereoscopic visual rendering.

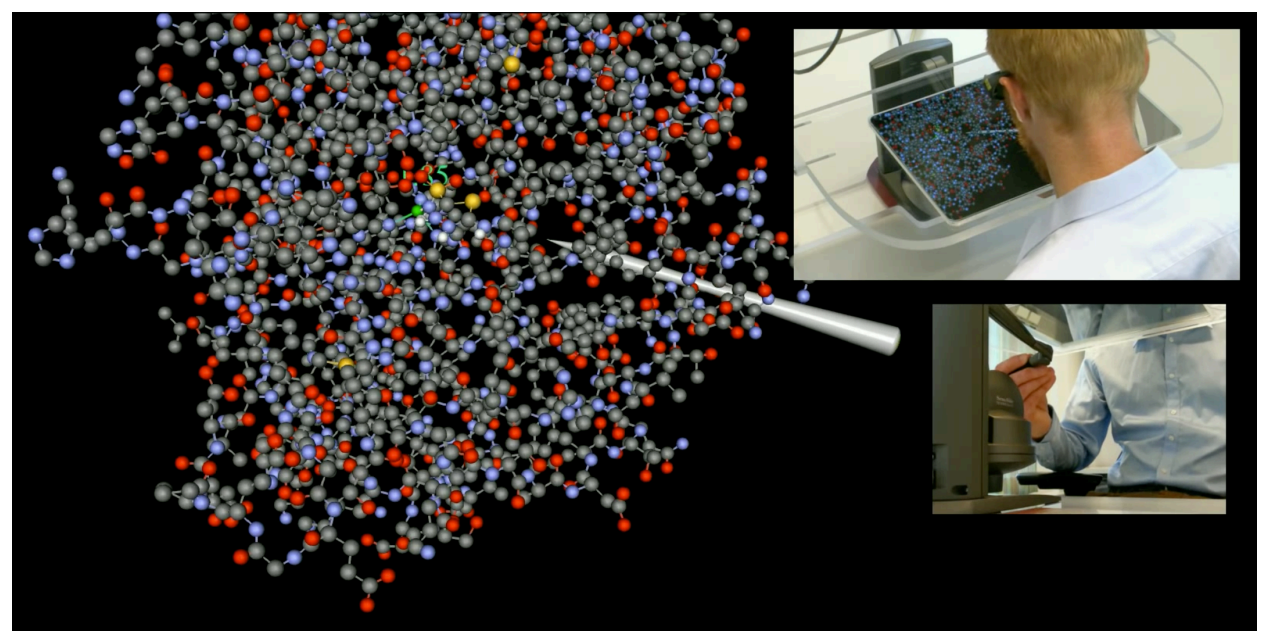

Figure 2. Haptic exploration of protein docking. The docking "force" is perceived through the haptic interaction and is collocated with visual stereoscopic images of the involved molecules.

This application was tested in several learning environments and at the Visualization Center. Through extensive evaluation, it was concluded that the haptic system helped students to learn more about the process of protein-ligand recognition. It also changed the way they reasoned about molecules to include more forcebased explanations. When the system was used by upper secondary students and the audience at the Visualization Center, they seemed to grasp the principles of the process. From an information-processing standpoint, visual and haptic coordination was seen to offload the visual pathway by placing less strain on visual working memory. From an embodied-cognition perspective, visual and tactile sensorimotor interactions may promote the construction of knowledge. The system may also have prevented students from drawing erroneous conclusions about the process. The results have cognitive and practical implications for the use of multimodal VR technologies in educational contexts. ${ }^{8} 9$

\section{Bringing an Audience to Space}

Following a knowledgeable guide on an interactive exploration of space is the dominant form of planetarium shows, from historical planetariums with analog "star ball" projectors to the introduction of digital planetariums in the 1990s. The development of computer graphics and visualization has paved the way for interactive and immersive experiences. An early example of such software was Uniview, ${ }^{10}$ which has been developed since 2003 and is now widely used in the planetarium community. A more recent example is OpenSpace, ${ }^{11} \mathrm{a}$ NASA-funded initiative in open source interactive data visualization software designed to visualize the entire known universe and portray our ongoing efforts to investigate the cosmos. It supports interactive, contextualized presentations of dynamic data from observations, simulations, and space mission planning and operations.

The versatility of OpenSpace has been used to create science communication events such as the one related to the passage of Pluto by the New Horizons spacecraft in 2015 (see Figure 3). In a live "domecast," 11 sites 
were connected. Visualization of the position and workings of the spacecraft was produced using OpenSpace, effectively letting the audience fly with the spacecraft toward Pluto. At the same time, mission experts were connected using videoconferencing to discuss the workings of the instruments, creating a "Science Live" experience. Another recent feature of OpenSpace is the ability to visualize planetary surfaces with extreme resolution. It is now possible to bring the audience to the surface of Mars in interactive exploration of the topography at $25-\mathrm{cm}$ resolution. ${ }^{12}$

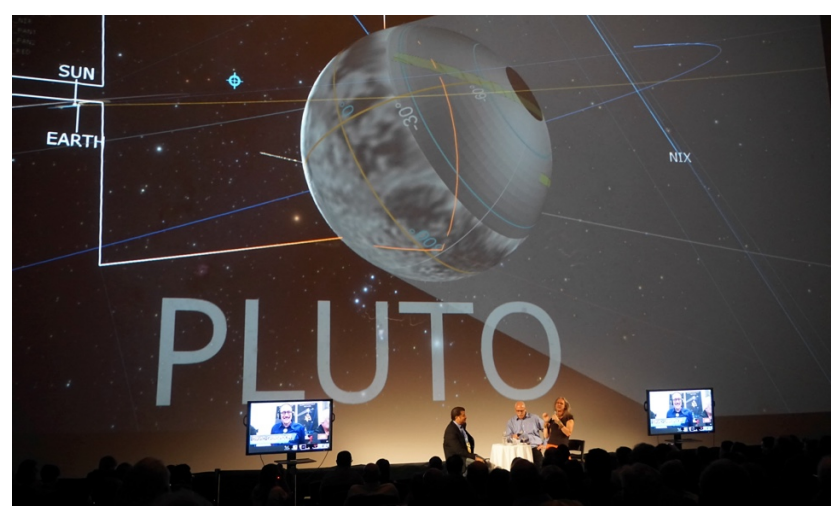

Figure 3. OpenSpace was used to visualize how the spacecraft New Horizons captured images of Pluto on 14 June 2015. In a live event, sites from all over the world were connected. The image shows the New York site with host Neil deGrasse Tyson.

With OpenSpace, our work has progressed in a new direction of mediated exploranation in large-scale immersive environments. We have found that the levels of audience engagement can be remarkably high, due mainly to the immediacy of the mediated, nonlinear exploration of more or less real-time data (as opposed to a preproduced movie). However, this requires significant skills and knowledge on behalf of the guide navigating space for the audience.

The OpenSpace collaboration will progressively include more features and data. In addition to advanced navigation interfaces, our current research agenda comprises implementation of tools to analyze data from the ongoing Gaia mission and improved support for dynamic, large-scale simulation data such as space weather and galaxy formation.

\section{DESIGN PRINCIPLES FOR EXPLORANATION}

Our work on exploranation in various settings has been largely explorative in itself. We have been feeling our way, designing visualizations and interactions based on a general sense of the data and the audience, identifying and elaborating the most successful ideas. In this kind of extended process, experience accumulates over time and eventually forms the basis for articulation and abstraction. This section summarizes our main insights in four design principles for exploranation that may be of value for other designers, engineers, and storytellers involved in science communication.

In interaction design in general, the task of designing explanations entails knowing the topic, the audience, and the available media. The responsibility lies with the designer to construct an explanation that fits the communicative situation. Designing for exploration, on the other hand, is a matter of setting the stage and providing the props for users to explore the topic at hand. The designer exerts indirect influence by defining the extent of the subject matter to be explored and the properties of the exploration tools provided. Designing for exploranation, where explanation and exploration converge, becomes largely a question of accommodating mixed-initiative interaction, as the following four principles demonstrate.

\section{Interleaving Explorative Microenvironments and Signposted Narratives}

An overall structure we find conducive to exploranation includes making curiosity-arousing parts of the data available for the users' self-directed exploration. Within those parts, annotations and other techniques are used to provide local explanatory information, also under the users' control. The explorative parts are linked together into an overall narrative structure, which is crafted in advance to tell a compelling story. 
In our interactive multitouch-table productions, for example, annotation callouts are used to focus the user's attention on the parts of the 3D volume that are the most rewarding ones for deeper exploration. On a higher narrative level, different sections of the interactive experience are presented within an overall structure where explanatory information is presented in a navigation interface to convey the big picture.

The guided space explorations exhibit similar narrative structures combining big-picture views (in a very literal sense!) and sweeping movement over great distances with close-up examinations of particular points of interest, such as the most detailed images of the Mars surface to ever be shown to the public. The framing of the presentation as a live event under the control of a knowledgeable guide makes the combination of big stories and in-depth examinations significantly more engaging than a prerecorded movie using the same narrative structure. From the audience point of view, a skilled performer can make the experience seem remarkably explorative even though there is no actual control of navigation or focus of attention.

\section{Constraining Explorative Interactions while Leveraging Pliability}

In each explorative microenvironment, the explorative tools are carefully constrained to convey a sense of expressiveness and agency to the users while at the same time ensuring that views resulting from manipulations are never disappointing or difficult to interpret. The interaction is highly responsive, with a tight connection between user actions and resulting outputs, thus forming a pliable user experience that is known to facilitate explorative behavior in interactive visualizations. ${ }^{13}$

The tools to rotate, crop, and dynamically light the 3D volume in our interactive multitouch-table productions illustrate the combination of productive constraints and pliability. They do not offer complete freedom of manipulating orientation and perspective, but rather nudge the user toward staying within visually interesting views of the most topical content. The MolDock example illustrates the didactic benefits of extending this principle to multimodal interaction. Similarly, the need for semantic-level navigation tools in the guided space explorations instantiates the principle of productive interaction constraints in an immersive environment (more on this later).

\section{Foregrounding the Topic through Perceptual Layering}

Exploranation is facilitated by keeping the object of interest in focus in a very concrete sense. Exploration tool palettes are relegated to the visual periphery. Annotations and other complementary information are visually subordinated to the topical objects. Icons and other parts of the supporting interface employ a subdued form and color scheme. This is a general principle in interaction and information design, and it is used consistently in the examples provided above.

\section{Supporting Performative Interaction}

Engaging with an interactive installation in a public space entails the tacit taking on of different roles by audience members. These roles can include a performer navigating the interaction, a number of viewers engaging in dialogue with the performer, and a number of bystanders gaining serendipitous insights and balancing between engaging more deeply and moving on. This is quite different from designing for generic multiuser interaction. ${ }^{14}$

In the case of interactive DVR, the interaction paradigm employed for the interactive touch tables epitomizes this principle with tools enabling an impromptu performer to take control through highly visible physical actions. It is commonplace in museums and galleries to observe a hotspot of interest and energy forming around one of our touch tables, where a visitor is exploring a dataset in the manner of performing for his or her group. The visibility and social inclusiveness of the explorative actions invite strangers into a temporary social structure forming around objects of mutual interest.

In the guided space explorations, the role of the guide is performative in a more conventional and formalized sense, taking the audience through the story. What is more, the visually immersive environment poses unique requirements on the performer, in the visceral sense of creating a pleasant trajectory through space. Camera movement and other interaction needs to be slow, smooth, and steady in order to avoid motion sickness and other discomfort. More research is needed to create semantic-level "virtual pilots" supporting the performer in such settings. 


\section{CONCLUSION}

Exploranation methodology is in its infancy, and further research is needed on technology, communicative strategies, and application domains to understand the possibilities and limitations. From a learning perspective, data-driven interactive visualization is a relatively new medium, with opportunities as well as challenges. It poses new and different requirements for the visualization tools and for how to guide the experience. In both cases, the aim is to instill in the user or audience a sense of curiosity and a desire to understand, while considering the diverse levels of previous knowledge in heterogeneous audiences ranging from schoolchildren, to the general public, to domain experts. It is essential to provide not only interactive modes of self-directed exploration but also intuitive and correct feedback. This requires adaptive interfaces as well as knowledgeable and sensitive guidance..$^{15-17}$

However, it is the story and how it is told that make the difference. Furthermore, among the skills needed for the guidance are the ability to simultaneously handle a wide range of preconceptions, adaptive storytelling skills, and knowledge of specific design principles for education, without causing cognitive overload. This article points to the need for research to meet these challenges - i.e., to integrate the cognitive and sociocultural perspectives and accommodate a diverse group of users in learning through exploranation.

\section{ACKNOWLEDGMENTS}

The authors wish to thank the Swedish eScience Research Center (SeRC) and NASA for support of the OpenSpace project. The support of the Swedish Research Council for funding of underpinning research is greatly acknowledged. The Knut and Alice Wallenberg Foundation is supporting the WISDOME project through a generous donation for development of dome productions and technology. The authors would also like to acknowledge the excellent support and contributions from the staff at the Norrköping Visualization Center $\mathrm{C}$ and the division for Media and Information Technology at Linköping University.

\section{REFERENCES}

1. C. Lundström, P. Ljung, and A. Ynnerman, "Local histograms for design of Transfer Functions in Direct Volume Rendering," IEEE Transactions on Visualization and Computer Graphics, vol. 12, no. 6, 2006, pp. 1570-1579.

2. E. Sundén, A. Ynnerman, and T. Ropinski. "Image plane sweep volume illumination," IEEE Transactions on Visualization and Computer Graphics, vol. 17, no. 12, 2011, pp. 2125-2134.

3. C. Lundström, T. Rydell, C. Forsell, A. Persson, and A. Ynnerman, "Multi-touch table system for medical visualization: Application to orthopedic surgery planning," IEEE Transactions on Visualization and Computer Graphics, vol. 17, no. 12, 2011, pp. 1775-1784.

4. A. Ynnerman, T. Rydell, D. Antoine, D. Hughes, A. Persson, and P. Ljung, "Interactive visualization of 3D scanned mummies at public venues," Communications of the ACM, vol. 59, no. 12, 2016, pp. 72-81.

5. K. Scalise, M. Timms, A. Moorjani, L.K. Clark, K. Holtermann, and S. Irvin, "Student Learning in Science Simulations: Design Features that Promote Learning Gains," Journal of Research in Science Teaching, Vol 48, no. 9, 2011, pp 1050-1078.

6. M. J. Jacobson, "Problem solving, cognition, and complex systems: Differences between experts and novices,". Complexity, vol. 6, no. 3, 2001, pp. $41-49$.

7. K. Lundin, M. Cooper, A. Persson, D. Evestedt, and A. Ynnerman, "Enabling design and interactive selection of haptic modes," Virtual Reality Journal, vol. 11, no. 1, 2007, pp. 1-13, 2007.

8. P. Bivall, S. Ainsworth and L.A.E. Tibell, "Do Haptic Representations Help Complex Molecular Learning?," Science Education, vol. 95, no. 4, 2011, pp. 700 - 719.

9. K. J. Schönborn, P. Bivall and L.A.E Tibell, "Exploring relationships between students' interaction and learning with a haptic virtual biomolecular model," Computers and education, vol. 57, no. 3, 2011, pp. 2095-2105.

10. S. Klashed, P. Hemingsson, C. Emmart, M. Cooper, and A. Ynnerman. "Uniview - Visualizing the Universe,”. In Eurographics 2010 - Areas Papers, Norrköping, Sweden, 2010, pp. 37-43.

11. A. Bock, E. Axelsson, K. Bladin, J. Costa, G. Payne, M. Territo, J. Kilby, M. Kuznetsova, C. Emmart, and A. Ynnerman, "Openspace: An open-source astrovisualization framework," Journal of Open Source Software, vo. 2, no. 15, 2017, pp 281. 
12. K. Bladin, E. Axelsson, E. Broberg, C. Emmart, P. Ljung, A. Bock, and A. Ynnerman, "Globe browsing: Contextualized spatio-temporal planetary surface visualization," IEEE Transactions on Visualization and Computer Graphics, vol. 24, no. 1, 2018, pp. 802-811.

13. J. Löwgren, "Pliability as an experiential quality: Exploring the aesthetics of interaction design," Artifact, vol. 1, no. 2, 2007, pp. 85-95.

14. P. Dalsgaard and L. K. Hansen, "Performing perception — staging aesthetics of interaction," $A C M$ Transactions on Computer-Human Interaction, vol. 15, no. 3, 2008, \#13.

15. D. Clark, E. Tanner-Smith and S. Kolloingsworth, "Digital games, Design and Learning: A Systematic review and Meta-Analysis", Review of Educational Research, vol. 86, no. 1, 2016, pp. 79122.

16. C. D'Angelo, D. Rutstein, C. Harris, R. Bernhard, E. Borokhovski and G. Haertel, "Simulations for STEM Learning: Systematic Review and Meta-Analysis," SRI International, 2014.

17. C. Zahn, "Digital Design and Learning: Cognitive-Constructivist Perspectives," In: Schwan S., Cress U. (eds) The Psychology of Digital Learning. Springer, Cham, 2017.

\section{ABOUT THE AUTHORS}

Anders Ynnerman holds the chair in scientific visualization at Linköping University, is the director of the Norrköping Visualization Center C, and is a research professor at the University of Utah. Contact him at anders@ynnerman.se.

Jonas Löwgren is professor of interaction and information design at Linköping University. Contact him at jonas.lowgren@liu.se.

Lena Tibell is a professor of biochemistry and visual learning and communication at Linköping University. Contact her at lena.tibell@liu.se.

Contact department editor Beatriz Sousa Santos at bss@ua.pt and department editor Ginger Alford at alfordg@trinityvalleyschool.org. 